Supplement of

\title{
Employing airborne radiation and cloud microphysics observations to improve cloud representation in ICON at kilometer-scale resolution in the Arctic
}

Jan Kretzschmar et al.

Correspondence to: Jan Kretzschmar (jan.kretzschmar@uni-leipzig.de)

The copyright of individual parts of the supplement might differ from the CC BY 4.0 License. 

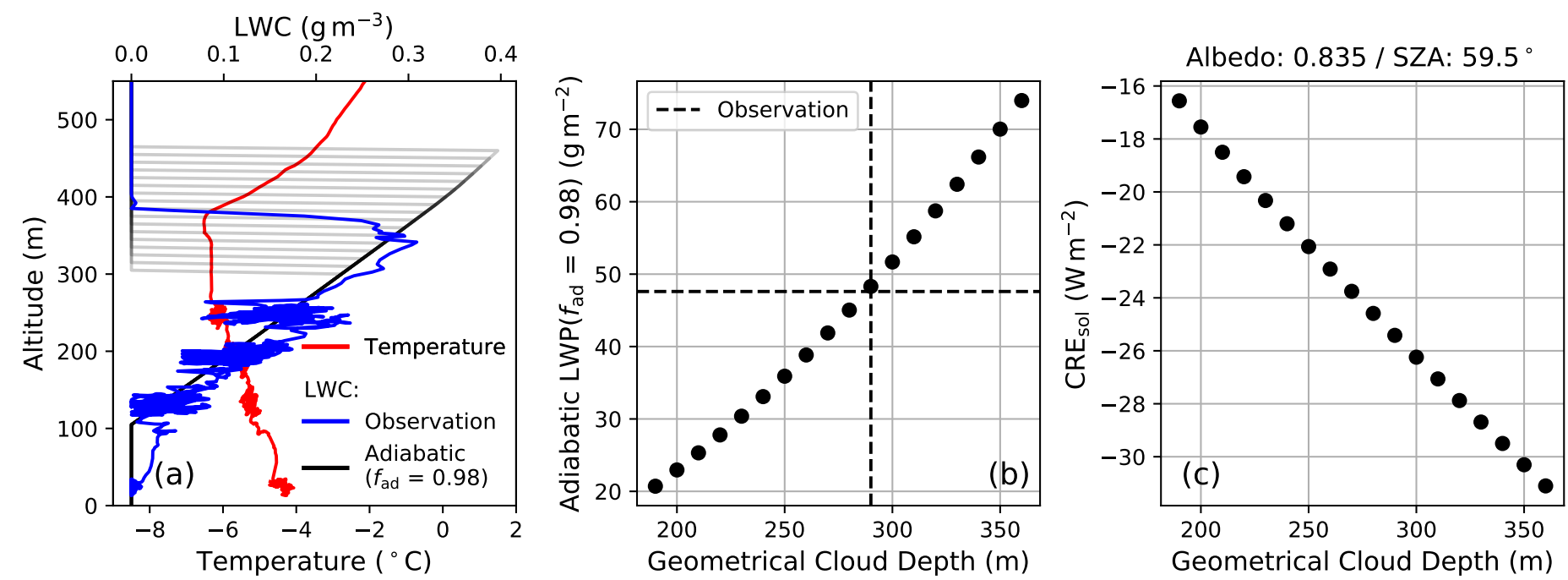

Figure S1: (a) Observed in-situ vertical profile of temperature (red) and liquid water content (blue) for vertical profile near R/V Polarstern on 4 June. The black line is the linearly interpolated LWC with an adiabaticity factor $f_{\text {ad }}$ of 0.98 .

(b) LWP as a function of geometrical cloud depth using with $f_{\mathrm{ad}}$ of 0.98 . The dashed lines indicate the observed geometrical cloud depth and LWP.

(c) $\mathrm{CRE}_{\mathrm{sol}}$ as a function of geometrical cloud depth. $\mathrm{CRE}_{\mathrm{sol}}$ has been derived from offline radiative transfer simulations using the respective LWP as calculated in (b). Deviation of $\mathrm{CRE}_{\text {sol }}$ compared to what has been observed by ACLOUD in the flight sections used in Figure S2 stem from lower albedo $(\sim 0.79)$ in these flight sections compared to the offline radiative transfer simulations $(0.835)$. 

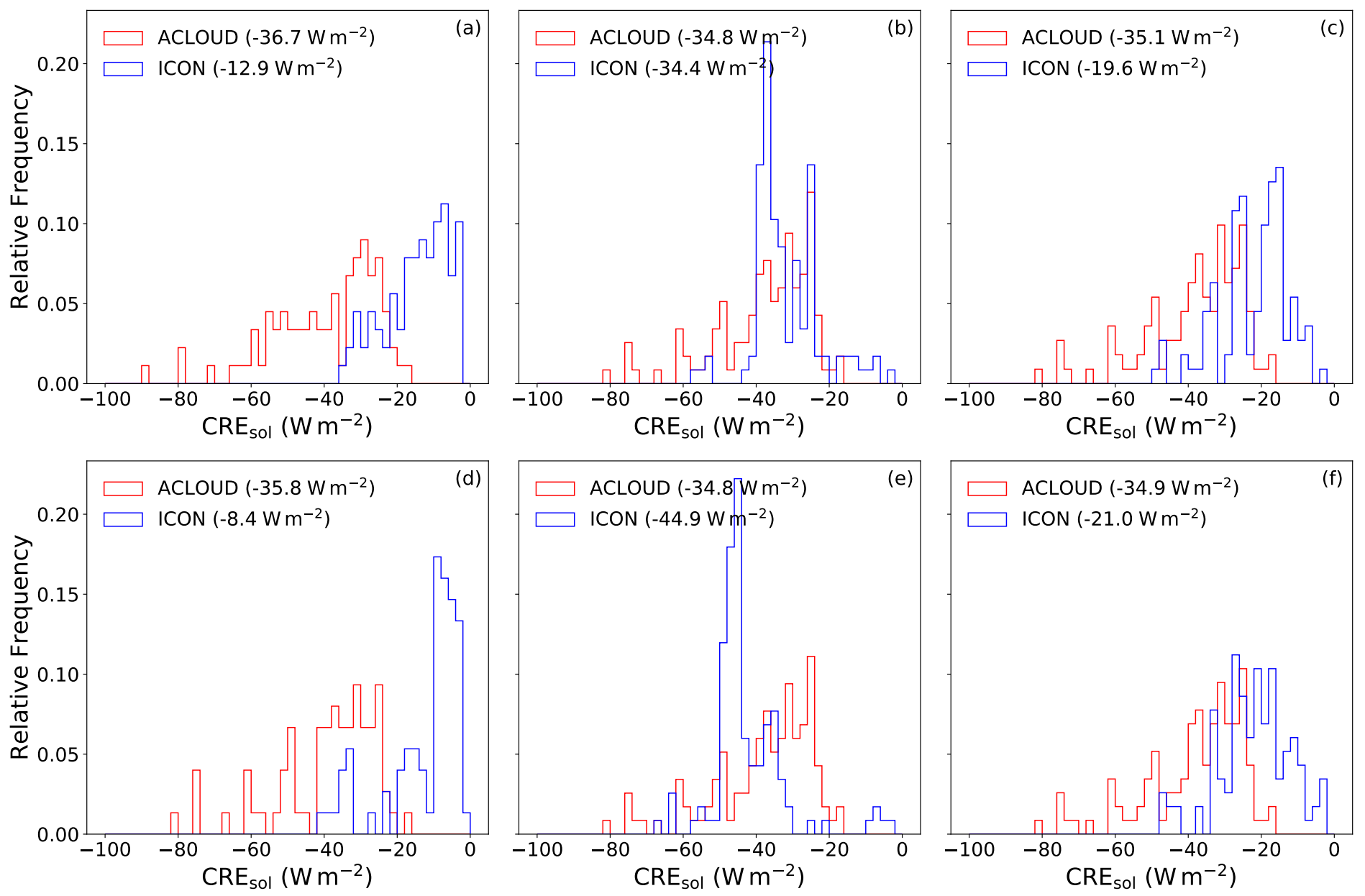

Figure S2: As Fig. 4b, but for the flights from 2 June to 5 June only, for the default set-up (a), for the revised CCN activation (b) and for the revised CCN activation with scaled number of activated CCN by a factor of 0.4 (c). The bottom row (d-f) as the top row but with hydrometeor number concentration coupled to radiation. Due to different cloud fields in the respective simulations, the histograms for the ACLOUD observations are not identical as only datapoints in the observations and the simulation are being used if both are within a cloud simultaneously. 

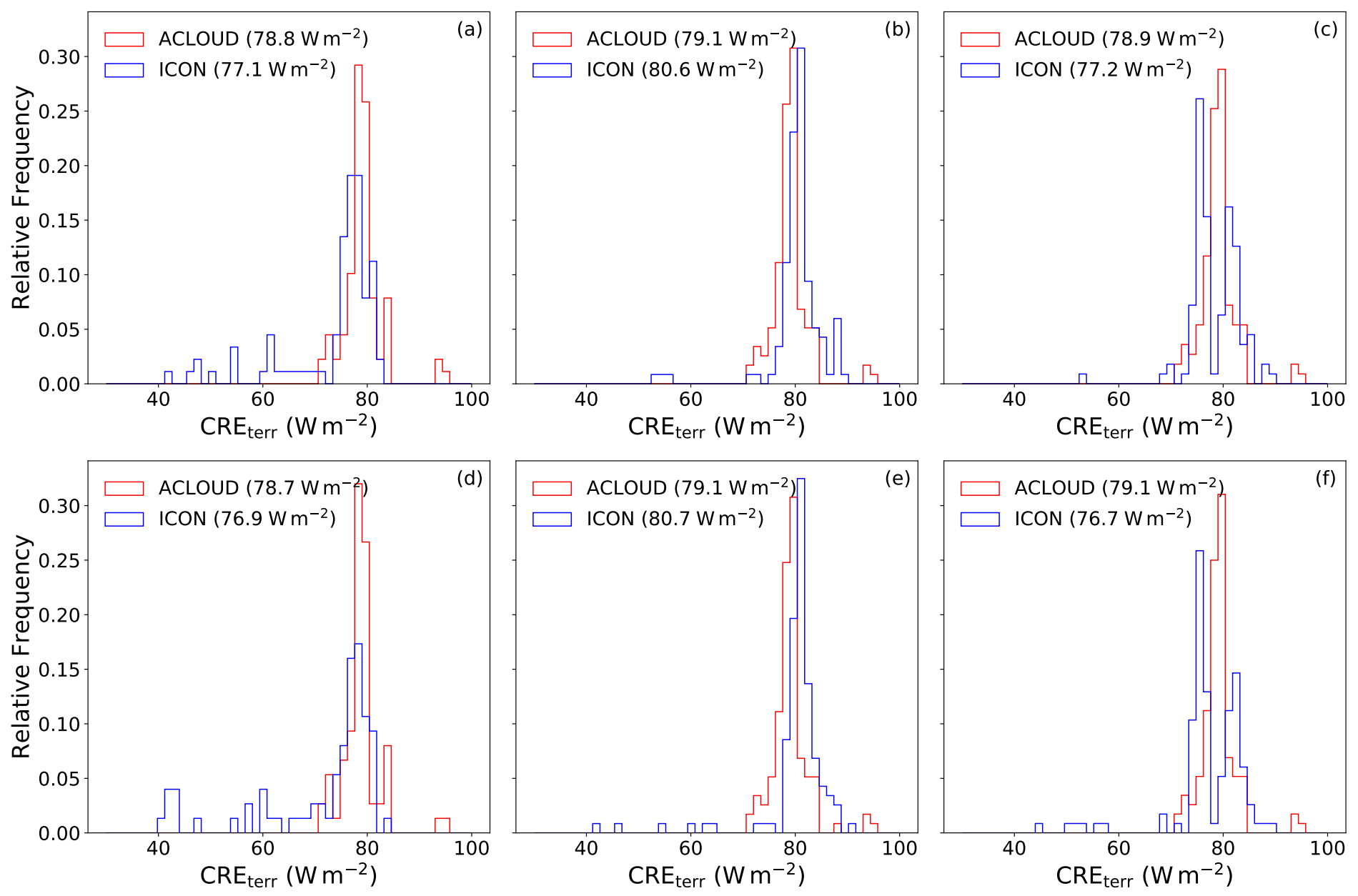

Figure S3: As Fig. 4c, but for the flights from 2 June to 5 June only, for the default set-up (a), for the revised CCN activation (b) and for the revised CCN activation with scaled number of activated CCN by a factor of 0.4 (c). The bottom row (d-f) as the top row but with hydrometeor number concentration coupled to radiation. Due to different cloud fields in the respective simulations, the histograms for the ACLOUD observations are not identical as only datapoints in the observations and the simulation are being used if both are within a cloud simultaneously. 

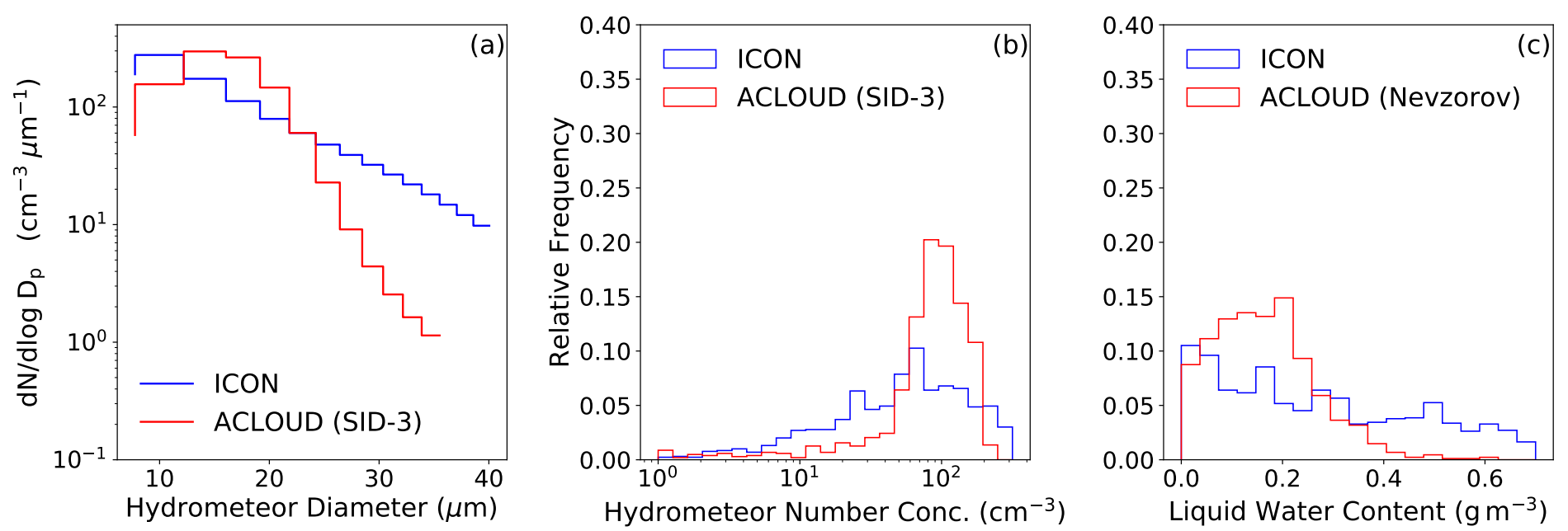

Figure S4: As Figure 6 but with autoconversion turned off. 